\title{
Exploring the Connection between Coherent Acoustic Phonons and Bright-Field Contrast in Ultrafast Electron Microscopy
}

\author{
Daniel X. Du ${ }^{1}$ and David J. Flannigan ${ }^{1 *}$ \\ 1. Department of Chemical Engineering and Materials Science, University of Minnesota, Minneapolis, \\ MN 55455, USA \\ * Corresponding author: flan0076@umn.edu
}

Intense ultrafast photoexcitation of archetypal semiconducting materials, such as Ge and GaAs, results in the rapid generation of large charge-carrier densities that propagate outward from the high-fluence region at initially hypersonic velocities. This produces a number of interesting effects that become interwoven with the underlying lattice, including the development of acoustic-type propagating oscillatory modes, excitation of coherent propagating strain waves, and coalescence of phase behaviors of the acoustic phonons and charge carriers via wave-particle drag. Using bright-field imaging in an ultrafast electron microscope [1], we directly imaged the behavior of photoexcited, nanoscale coherent strain waves in single-crystal Ge [2,3]. In addition to single preferential wavevectors and initially hypersonic phase velocities (up to $35 \mathrm{~nm} / \mathrm{ps}$ ), we were able to directly resolve single-phonon constant velocities and an overall time-varying phase-velocity dispersion to the bulk speed of sound over approximately one nanosecond. Comparison to dispersion behaviors expected for symmetric and asymmetric Lamb-type modes showed good agreement with experiments, suggesting the preferential excitation of a single, symmetric first-order mode. However, quantitative comparison to the expected and measured contrast strengths associated with the coherent dynamics is needed in order to further elucidate the precise microscopic mechanisms.

Here, we present the results of kinematic two-beam and multi-beam diffraction simulations coupled to calculated Lamb-wave distortions of a single mode reaching maximum strains of $1 \%$. In essence, the simulations treat the lattice distortions arising from strain associated with the coherent structural dynamics as time-varying oscillations in the local Bragg-scattering condition. For the two-beam condition, we find the photoinduced strains alone cannot account for the experimentally-observed contrast strengths. Indeed, the simulated bright-field images show an almost complete absence of any coherent contrast-strength modulation. The multi-beam condition returns similar results, though static contrast bands arise due to Bragg-spot interference. Direct comparisons of the simulated and experimental contrast strengths for the pure-strain condition reveal a significant discrepancy, with the observed strengths associated with the coherent dynamics being much higher than the simulated strengths. We identify a number of possible sources of the discrepancy, including variations in the incident electron wavevector, the existence of phase contrast and multiple scattering from dynamical diffraction, the presence of large transient strains wherein dislocation motion does not have time to occur, and additional contributions to the loss in transmitted intensity due to additional scattering from photoexcited charge carriers. This last possible effect is especially intriguing, as the effects of photoexcited charge carriers on the elastic scattering of high-energy electrons has been little explored. Indeed, in light of the previous observations noted above, fluctuations in the local electrostatic potential in the lattice may have a significant effect on the intensity at areas of higher strain and therefore higher carrier concentration [4]. 
[1] DA Plemmons, PK Suri and DJ Flannigan, Chem. Mater. 27 (2015), p. 3178.

[2] DR Cremons, DX Du and DJ Flannigan, Phys. Rev. Mater. 1 (2017), p. 073801.

[3] DR Cremons, DA Plemmons and DJ Flannigan, Nat. Commun. 7 (2016), p. 11230.

[4] This material is based on work supported by the U.S. Department of Energy, Office of Science,

Office of Basic Energy Sciences under Award No. DE-SC-0018204.
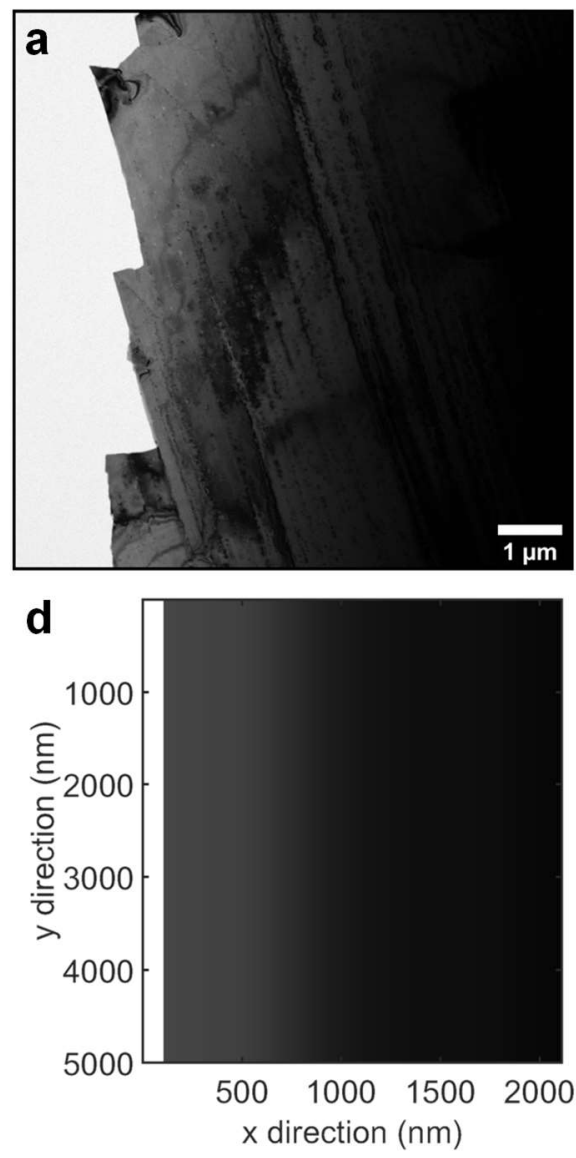
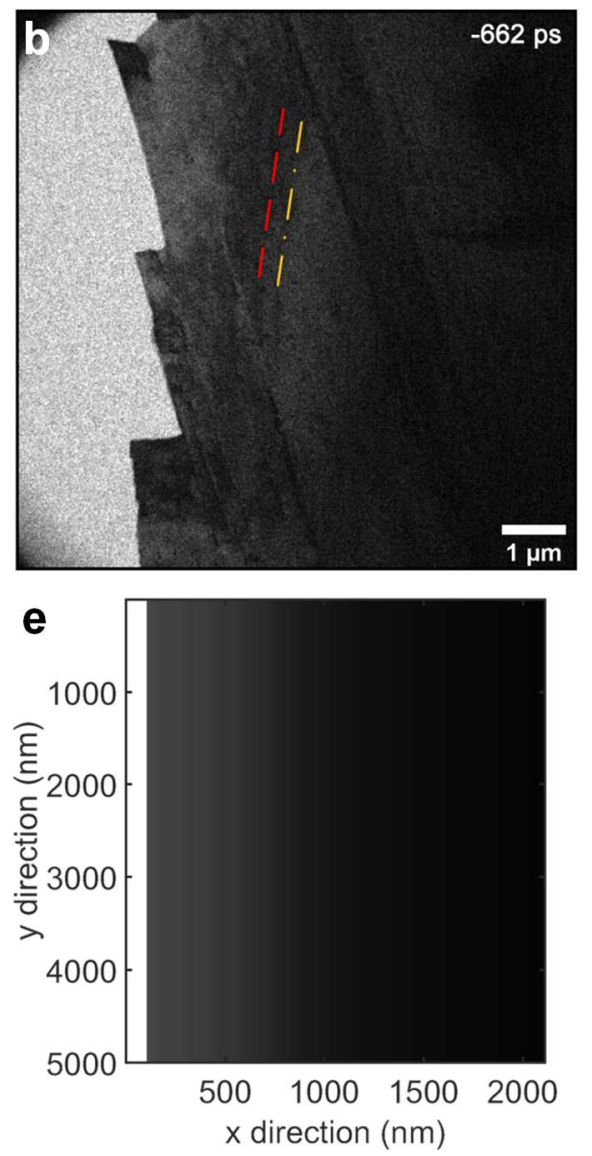
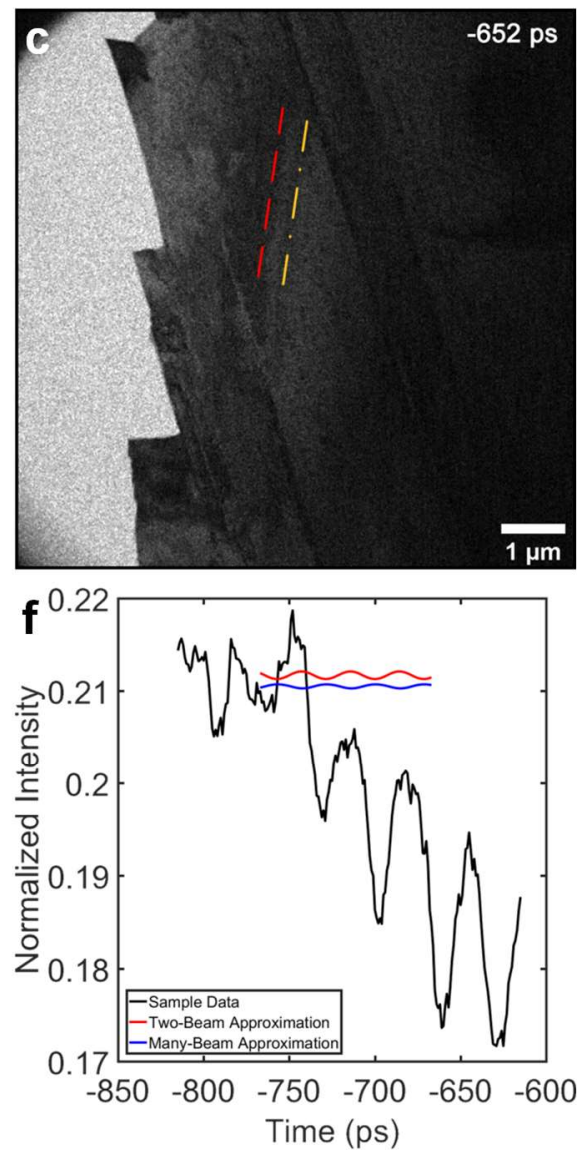

Figure 1. Comparison of simulated $4^{\circ}$ Ge wedges under first-order symmetric Lamb-type strain to acquired UEM contrast data. (a) Bright-field TEM image of the $4^{\circ}$ Ge wedge studied with UEM. (b) A representative UEM bright-field image acquired with a delay-stage position of $-662 \mathrm{ps.} \mathrm{The} \mathrm{time} \mathrm{is}$ arbitrary and not with respect to photoexcitation or any specific dynamics. The red and yellow markers note the positions of a reference and wavefront-analysis region, wherein the phonon propagation behaviors are extracted. (c) A UEM image acquired 10 ps after the image in (b). Note the position of the yellow marker, which marks the precise location of a single wavefront, has clearly changed relative to that in the previous image. (d) Top-down view of the $4^{\circ}$ Ge specimen simulated with a two-beam condition. The $x$-direction is into the bulk of the wedge along the direction of increasing thickness, while the $y$-direction is parallel to the vacuum-crystal interface. (e) Multi-beam simulated image using 400 Bragg spots surrounding the direct beam along the [110] zone axis. The orientation is the same as in (d). (f) Comparison of the measured and simulated contrast strengths arising from the ultrafast coherent strain waves showing the large discrepancy between experiment and the pure-strain calculations. 